Pacific Journal of Mathematic 


\title{
SOBOLEV APPROXIMATION BY A SUM OF SUBALGEBRAS ON THE CIRCLE
}

\author{
John B. Garnett AND ANThony G. O'FARRELL
}

Let $\psi$ be an orientation-reversing homeomorphism of the unit circle onto itself. We consider approximation in certain Sobolev norms by functions of the form $f(z)+g(\psi)$, where $f$ and $g$ are polynomials. The methods involve conformal welding and Hardy space theory. We construct a Jordan arc of positive continuous analytic capacity such that the harmonic measures for the two complementary domains are mutually absolutely continuous.

Let $C(S)$ denote the space of continuous, complex-valued functions on the unit circle $S$. For $f \in C(S)$, let $P(f)$ denote the space of all polynominals in $f$ with complex coefficients. Let $z$ denote the identity function. Browder and Wermer proved the following theorem [3, p. 551].

Let $\psi$ be a direction-reversing homeomorphism of $S$ onto $S$. Then the vector space sum $P(z)+P(\psi)$ is uniformly dense in $C(S)$.

In the first part of this paper we prove a result that partially extends this theorem to the $C^{1}$ norm. We say that a direction-reversing homeomorphism $\psi: S \rightarrow S$ is an involution if $\psi \circ \psi=z$. Let $C^{1}(S)$ denote the space of continuously-differentiable, complex-valued functions on $S$, with the norm

$$
\|f\|_{C^{1}}=\|f\|_{\infty}+\left\|\frac{d f}{d \theta}\right\|_{\infty} .
$$

If $\alpha>0$, let $C^{1+\alpha}(S)$ denote the space of functions $f$ in $C^{1}(S)$ such that $f^{\prime}=d f / d \theta$ satisfies a Lipschitz condition with exponent $\alpha$ :

$$
\left|f^{\prime}(a)-f^{\prime}(b)\right| \leqq K|a-b|^{\alpha}
$$

for all points $a$ and $b$ in $S$.

THEOREM. Let $\alpha>0$, and let $\psi \in C^{1+\alpha}(S)$ be an involution. Then $P(z)+P(\psi)$ is dense in $C^{1}(S)$.

Our proof of this theorem is radically different from Browder and Wermer's proof of their result. By means of duality and conformal welding, we reduce the theorem to a statement about 
removable singularities for a certain Hardy class, which we then prove.

For $p \geqq 1$, let $W^{p}$ denote the Sobolev space

$$
\left\{f \in C(S): \frac{d f}{d \theta} \in L^{p}\left(d_{\theta}\right)\right\} \text {. }
$$

The norm on the algebra $W^{p}$ is

$$
\|f\|_{W^{p}}=\|f\|_{\infty}+\left\|\frac{d f}{d \theta}\right\|_{p} .
$$

In the second part of the paper we construct an involution $\psi \in W^{1}$ such that the sum $P(z)+P(\psi)$ is not dense in $W^{1}$. This same $\psi$ provides the answer to a problem raised by Wermer. He asked for an absolutely-continuous involution $\psi$ such that there exists a nonconstant function $f$, analytic on the open unit disc $D$, and continuous on $D \cup S$, such that $f \circ \psi=f$ on $S$.

We wish to thank John Wermer for suggesting the problem discussed here. The idea of using welding in this way is due to Wermer. We are grateful to Lennart Carleson for suggesting the argument of section (1.4).

1. Proof of the theorem.

1.1. Let $\alpha>0$, and let $\psi \in C^{1+\alpha}(S)$ be an involution. We wish to prove that $P(z)+P(\psi)$ is dense in $C^{1}(S)$. Each linear fractional transformation that fixes $D$ is approximable in $C^{1}(S)$ by polynominals. Hence we may assume that the fixed points of $\psi$ are -1 and 1 .

Clearly, $P(z)+P(\psi)$ is dense in $C^{1}(S)$ if and only if $P(z)+\psi^{\prime} P(\psi)$ is dense in $C(S)$ (in the uniform norm). By a duality argument and the F. and M. Riesz theorem, this happens if and only if

$$
H^{1}(z) \cap H^{1}(\psi)=C,
$$

where $H^{1}(z)$ denotes the usual Hardy class on the circle (that is, $H^{1}(z)$ consists of the functions $f \in L^{1}(d \theta)$ such that $\int f z^{n} d \theta=0, n=1,2$, $3, \cdots), H^{1}(\psi)$ denotes

$$
\left\{f \circ \psi: f \in H^{1}(z)\right\},
$$

and $C$ denotes the constant functions.

To finish the proof, we need to prove (1).

1.2. Fix $f \in H^{1}(z) \cap H^{1}(\psi)$. Since $\psi$ is an involution, the functions

$$
g=\frac{1}{2}(f+f \circ \psi),
$$




$$
h=\frac{1}{2}(f-f \circ \psi)
$$

belong to $H^{1}(z)$, and furthermore

$$
g \circ \psi=g, \quad h \circ \psi=-h,
$$

almost everywhere on $S$.

We claim that there exists a $C^{1+\alpha / 2}$ are $\Gamma$ on the Riemann sphere $\Sigma$, and a conformal map $\varphi: D \rightarrow \Sigma \sim \Gamma$, such that the continuous extension of $\varphi$ to $S$ satisfies $\varphi \circ \psi=\varphi$ on $S$. By a $C^{1+\alpha / 2}$ arc we mean the image of the closed unit interval under some $C^{1+\alpha / 2}$ injective map with non-vanishing derivative.

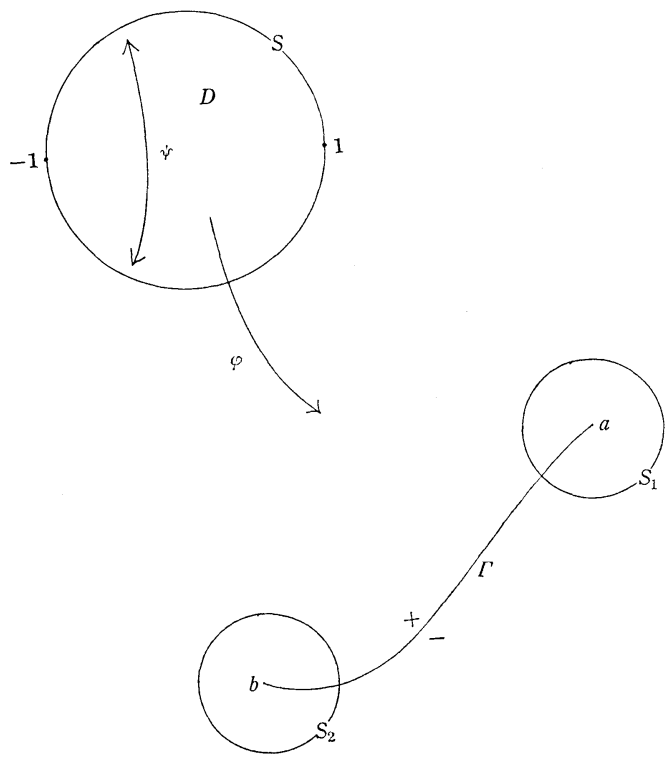

Figure 1.

To prove this claim it is sufficient to rewrite the classical welding argument [6, 7], keeping track of the smoothness of the various functions involved. The Beurling-Ahlfors solution [2, p. 135, equation (14)] of the quasi-conformal extension problem yields a $C^{1+\alpha}$ extension, given $C^{1+\alpha}$ data. Bers has proved [1] that the solution $F$ of the Beltrami equation

$$
\begin{aligned}
\frac{\partial F}{\partial \bar{\zeta}}(\zeta) & =G(\zeta) \frac{\partial F}{\partial \zeta}(\zeta), \quad(\zeta \in C) \\
F(\infty) & =\infty,
\end{aligned}
$$

belongs to $C^{1+\alpha}(C)$ if $G$ belongs to $\operatorname{Lip}(\alpha, C)$, and $\|G\|_{\infty}<1$. So the welding argument [6, Lemma 1, Lemma 3, Theorem 1] shows that 
$\Gamma$ exists, and is locally $C^{1+\alpha}$, except possibly at the endpoints. A special argument is needed for the endpoints (make a preliminary map of the disc to the complement of the negative axis, and use the fact that $\left.\psi^{\prime}(1)=\psi^{\prime}(-1)=1\right)$, and the conclusion is that $\Gamma$ must be $C^{1+\alpha / 2}$ near these points. We also find that, to second order,

$$
\varphi(\zeta) \cong a+c(1-\zeta)^{2}
$$

for small $|\zeta-1|$, and

$$
\varphi(\zeta) \cong b+d(1+\zeta)^{2}
$$

for small $|\zeta+1|$, where $a, b$, $c$, and $d$ are certain complex constants.

\subsection{Define}

$$
g_{1}(\omega)=g\left(\varphi^{-1}(\omega)\right), \quad h_{1}(\omega)=h\left(\varphi^{-1}(\omega)\right),
$$

whenever $\omega \in \Sigma \sim \Gamma$. Then $g_{1}$ and $h_{1}$ belong to the Hardy class $H^{1}(\Sigma \sim \Gamma)$ [6]. In view of [5, p. 169, Corollary], it is clear that $H^{1}(\Sigma \sim \Gamma)$ is contained in the class $E^{1}(\Sigma \sim \Gamma)$. Hence $g_{1}$ and $h_{1}$ have normal limits from either side at almost all points of $\Gamma$, and the various limit functions are integrable with respect to arc length. Let us denote the limit functions by $g_{1}^{+}, g_{1}^{-}, h_{1}^{+}$, and $h_{1}^{-}$. We have

$$
g_{1}^{+}=g_{1}^{-}, \quad h_{1}^{+}=-h_{1}^{-}
$$

almost everywhere on $\Gamma$. An application of Morera's theorem shows that $g_{1}$ extends analytically across $\Gamma$, and hence is constant. (To do the argument at the endpoints simply continue $\Gamma$ a little further.) A similar argument shows that on any small disc $\Delta$, separated by $\Gamma$ into $U^{+}$and $U^{-}$, the function $h_{1}\left|U^{+},-h_{1}\right| U^{-}$extends analytically across $\Gamma \cap \Delta$. Hence $h_{1}^{2}$ extends analytically to $\Sigma \sim\{a, b\}$, where $a$ and $b$ are the endpoints of $\Gamma$.

1.4. Since $h \in H^{1}(z),\left(h(\zeta)=0\left((1-|\zeta|)^{-1}\right)\right.$ for $|\zeta|<1$, and since $h_{1}^{2}$ is analytic on $S_{1}^{2} \mid\{a, b\}$, we see from (2) and (2') that there is a constant $\kappa_{1}>0$ such that,

$$
\left|h_{1}(\omega)\right| \leqq \kappa_{1} \operatorname{dist}(\omega, \Gamma)^{-2},
$$

whenever $\omega \in \Sigma \sim \Gamma$. Thus there exists a constant $\kappa_{2}>0$ such that, for all $\rho$ with $0<\rho<|a-b| / 2$ we have the bound

$$
\int_{S_{i}}\left|h_{1}(\omega)\right|^{1 / 4}|d \omega| \leqq \kappa_{2} \rho^{1 / 2}
$$

for $i=1,2$, where

$$
\begin{aligned}
& S_{1}=\{\omega:|\omega-a|=\rho\}, \\
& S_{2}=\{\omega:|\omega-b|=\rho\} .
\end{aligned}
$$


Let $\mu_{\xi}$ denote harmonic measure for the point $\xi$ on $S_{1} \cup S_{2}$ with respect to

$$
\Sigma \sim\{\zeta:|\zeta-a| \leqq \rho \text { or }|\zeta-b| \leqq \rho\}
$$

Suppose

$$
0<\rho<|a-b| / 4 \text {, and }|\xi-a|=2 \rho \text {. }
$$

Then

$$
d \mu_{\xi}(\omega) \leqq 3 \rho^{-1}|d \omega|,
$$

and since $\left|h_{1}\right|^{1 / 4}$ is subharmonic,

$$
\left|h_{1}(\xi)\right|^{1 / 4} \leqq \int\left|h_{1}\right|^{1 / 4} d \mu_{\xi} \leqq 6 \kappa_{2} \rho^{-1 / 2}
$$

Hence,

$$
\left|h_{1}(\omega)\right| \leqq 6^{4} \kappa_{2}^{4} \max \left\{|\omega-a|^{-2},|\omega-b|^{-2}\right\}
$$

whenever $\omega \neq a$ or $b$. Thus $a$ and $b$ are at worst poles of $h_{1}^{2}$.

If $h_{1}^{2}$ had a pole at $a$, then $h(\zeta)$ would have growth at least $|1-\zeta|^{-1}$ at 1 , and this is impossible, since $h \in L^{1}(d \theta)$. Thus $h_{1}^{2}$ extends analytically over $a$, and similarly over $b$. Hence $h_{1}^{2}$ is constant, $h_{1}$ is constant, $h$ is constant, and $f=g+h$ is constant. We are done.

1.5. The obvious question is whether $P(z)+P(\psi)$ is dense in $C^{1}(S)$ whenever $\psi \in C^{1}(S)$ is a direction-reversing homeomorphism. We do not know. A result with a somewhat weaker hypothesis than our theorem is the following.

If $1 \leqq p \leqq 2$, if $\psi \in C^{1}(S)$ is an involution, and if there exists $\kappa>0$ such that

$$
\int\left|\frac{\psi^{\prime}(\theta)-\psi^{\prime}(\varphi)}{\theta-\phi}\right| d \theta \leqq \kappa
$$

for all $\varphi$, then $P(z)+P(\psi)$ is dense in $W^{p}$.

Proof. It suffices to prove the case $p=2$.

The sum $P(z)+P(\psi)$ is dense in $W^{2}$ if and only if the sum $P(z)+\psi^{\prime} P(\psi)$ is dense in $L^{2}$, and this happens if and only if

$$
H^{2}(z) \cap H^{2}(\psi)=C,
$$

where $H^{2}(z)$ is the usual Hardy space on the circle, and

$$
H^{2}(\psi)=\left\{f \circ \psi: f \in H^{2}(z)\right\}
$$


Now weld, as in the proof of the theorem. Volkoviskii [7] has shown that the welded arc $\Gamma$ is Lipschitzian (his argument has to be modified a little to cover the endpoints).

Let $f \in H^{2}(z) \cap H^{2}(\psi)$, and form $g, h, g_{1}$, and $h_{1}$ as before. The functions $g_{1}$ and $h_{1}$ lie in $H^{2}(\Sigma \sim \Gamma)$, hence $g_{1}$ and $h_{1}^{2}$ belong to $H^{1}(\Sigma \sim \Gamma)$. Since $\Gamma$ is Lipschitz, $g_{1}$ and $h_{1}^{2}$ have normal limits almost everywhere on $\Gamma$, from either side, the limit functions are integrable with respect to arc length on $\Gamma$, and the limits of each function from either side agree almost everywhere. Applying Morera's theorem, we conclude that $g_{1}^{2}$ and $h_{1}$ extend analytically across $\Gamma$, and hence are constant. Hence $f$ is constant.

2. The Example.

2.1. Let $\psi \in W^{1}$. Then $P(z)+P(\psi)$ is dense in $W^{1}$ if and only if $P(z)+\psi^{\prime} P(\psi)$ is dense in $L^{1}$. If there exists a nonconstant function $f \in C(S) \cap H^{\infty}(z)$ such that $f=f \circ \psi$, then $f$, regarded as an element of $L^{\infty}=\left(L^{1}\right)^{*}$, annihilates $P(z)+\psi^{\prime} P(\psi)$, hence $P(z)+P(\psi)$ is not dense in $W^{1}$.

2.2. Let $\Gamma$ be an arc on $\Sigma$, and let $\psi$ be the involution on $S$ induced by the conformal map $\varphi: D \rightarrow \Sigma \sim \Gamma$. Suppose $\varphi$ maps 1 and -1 to the endpoints of $\Gamma$, and $\varphi(0)=\alpha$, say. Then harmonic measure with respect to $\alpha$ for $\Sigma \sim \Gamma$ on $\Gamma$ may be regarded as made up of two pieces, $\mu^{+}$and $\mu^{-}$, where $\mu^{+}$is the image under $\varphi$ of $d \theta / 2 \pi$ measure on the upper half-circle, and $\mu^{-}$is the image of $d \theta / 2 \pi$ measure on the lower half-circle. The map $\psi$ belongs to $W^{1}$ if and only if $\mu^{+}$and $\mu^{-}$are mutually absolutely-continuous. If $F$ is a nonconstant analytic function on $\Sigma \sim \Gamma$ that extends continuously to $\Gamma$, then $f=F \circ \varnothing$ is analytic on $D$, continuous up to $S$, and satisfies $f \circ \psi=f$.

So what we wish to construct is an are $\Gamma \subset \Sigma$ such that the continuous analytic capacity of $\Gamma$ is positive, and the harmonic measures for the two sides (i.e. $\mu^{+}$and $\mu^{-}$) are mutually absolutelycontinuous.

LEMMA 2.3. Let $\gamma$ be a simple closed Jordan curve, enclosing a region $U$. Let $\Gamma$ be a Jordan arc that separates $U$ into two nonempty Jordan regions $V$ and $V^{\prime}$. Let $\alpha$ and $\beta$ be closed subsets of $\gamma \cap \operatorname{clos} V$, both at positive distance from $\Gamma$. For $a \in U$, let $\omega_{U}(a, E)$ denot the harmonic measure of $E$ with respect to $U$, evaluated at a. Suppose $\alpha$ and $\beta$ have positive harmonic measure with respect to $U$. Then there exists a constant $\kappa \geqq 1$ such that

$$
\frac{1}{\kappa} \leqq \frac{\omega_{U}(\alpha, \alpha)}{\omega_{U}(\alpha, \beta)} \leqq \kappa
$$


whenever $a \in V^{\prime}$. Moreover, the constant $\kappa$ may be chosen to depend on $V, \alpha$, and $\beta$, and not on the shape or size of bdy $V^{\prime} \sim \Gamma$.

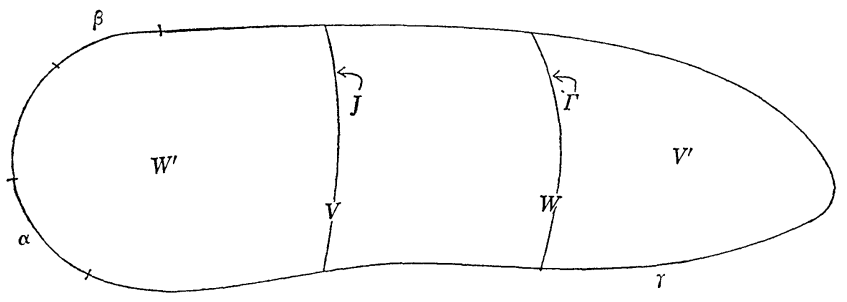

Figure 2.

Proof. Let $J$ be a Jordan arc that separates $U$ into two Jordan domains $W$ and $W^{\prime}$, such that $\alpha \cup \beta \subset$ bdy $W^{\prime}, U \cap J \subset V, U \cap \Gamma \subset W$, and $\operatorname{dist}(J, \Gamma \cup \alpha \cup \beta)>0$.

Pick a point $p \in$ bdy $V \sim(\alpha \cup \beta \cup \Gamma \cup J)$, and map $V$ conformally to the upper half-plane, so that $p$ goes to $\infty$. By comparing the Poisson kernel on the images of $\alpha, \beta$, and $\Gamma$, we see that there exist constants $\kappa_{1}$ and $\kappa_{2}$, depending only on $V, \alpha, \beta$, and $J$, such that

$$
\begin{aligned}
& \omega_{V}(a, \alpha) \leqq \kappa_{1} \omega_{V}(a, \beta), \\
& \omega_{V}(a, \Gamma) \leqq \kappa_{2} \omega_{V}(a, \beta),
\end{aligned}
$$

for all $a \in J \cap U$.

Hence, for $a \in J$ we have

$$
\begin{aligned}
\omega_{U}(\alpha, \alpha) & \leqq \omega_{V}(\alpha, \alpha)+\omega_{V}(\alpha, \Gamma) \\
& \leqq\left(\kappa_{1}+\kappa_{2}\right) \omega_{V}(\alpha, \beta) \\
& \leqq\left(\kappa_{1}+\kappa_{2}\right) \omega_{U}(\alpha, \beta) .
\end{aligned}
$$

By the maximum principle,

$$
\omega_{U}(\alpha, \alpha) \leqq\left(\kappa_{1}+\kappa_{2}\right) \omega_{U}(\alpha, \beta)
$$

for all $a \in W$. The reverse inequality is similar.

\subsection{The construction.}

The basic idea is to pass an arc $\Gamma$ through a Cantor set $C$ with positive continuous analytic capacity, in such a way as to ensure that $C$ has harmonic measure zero with respect to $\Sigma \sim \Gamma$, while $\Gamma \sim C$ is a union of line segments.

Let $C$ be the product of Cantor's tertiary set with itself. Then $C=\bigcap_{n=0}^{\infty} C_{n}$, where $C_{n}$ consists of $4^{n}$ squares of side $3^{-n}$. Denjoy [4] has shown that $C$ has continuous analytic capacity.

Let $\Gamma_{0}=C_{0}$. 
Let $\Gamma_{1}$ be the union of the four solid squares in $C_{1}$ and the three line segments shown in Figure 3.
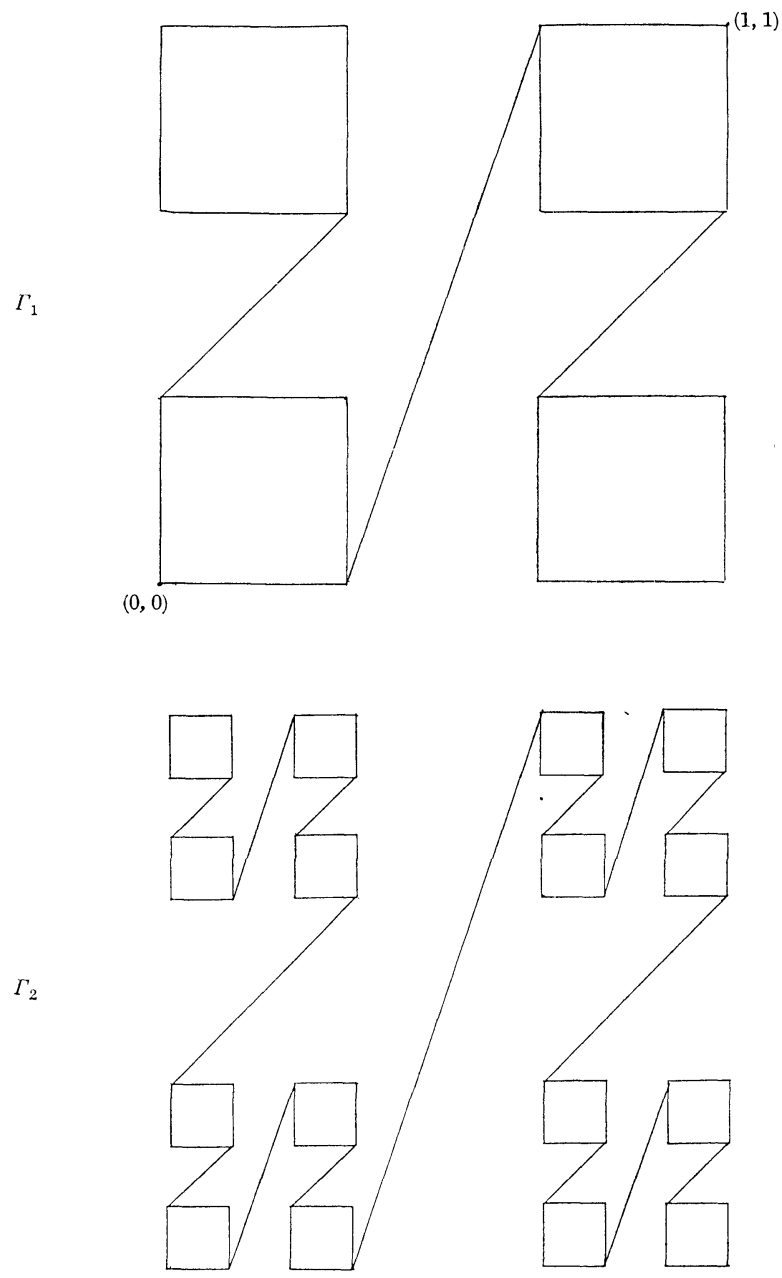

Figure 3.

Let $\Gamma_{2}$ be obtained from $\Gamma_{1}$ by leaving the three line segments alone, and replacing the four squares by four copies of $\Gamma_{1}$, each one reduced in scale by one-third, as in Figure 3.

Let $\Gamma_{n+1}$ be obtained from $\Gamma_{n}$ in the analogous way.

Then $\left\{\Gamma_{n}\right\}$ is a decreasing sequence of compact connected sets, and $\Gamma=\bigcap_{n} \Gamma_{n}$ is a Jordan arc that contains $C$. If $\mu_{+}=\mu_{+}+\mu_{-}$is harmonic measure for $\Sigma \sim \Gamma$, then $\mu_{+}$and $\mu_{-}$are mutually absolutelycontinuous with respect to arc length on the union of line segments $\Gamma \sim C$. Thus, to prove that $\mu_{+}$and $\mu_{-}$are mutually absolutelycontinuous it suffices to show that $\mu_{+}(C)=\mu_{-}(C)=0$.

Let $\gamma$ denote the union of $\Gamma$ with 


$$
\tau=\{x+i y \in C: x+y=1, x \leqq 0 \text { or } y \leqq 0\} .
$$

Then $\gamma$ separates the plane into two domains. Let $U$ be that domain to which the point 2 belongs. To prove that $\mu_{+}(C)=0$, it suffices to prove that $\omega(2, C)=0$, where $\omega$ is harmonic measure with respect to $U$.

Let $\omega^{(n)}(\alpha, E)$ be the harmonic measure for the subdomain of $U$ bounded by $\tau \cup \Gamma_{n}$. By applying Lemma 2.3 to six different choices of $(V, \alpha, \beta)$ we see that there is a constant $\kappa$ so that

$$
\omega^{(n+1)}\left(2, C_{n+1}\right) \leqq \kappa \omega^{(n+1)}\left(2, C_{n} \sim C_{n+1}\right) .
$$

But

$$
\omega^{(n+1)}\left(2, C_{n+1}\right)+\omega^{(n+1)}\left(2, C_{n} \sim C_{n+1}\right) \leqq \omega^{(n)}\left(2, C_{n}\right)
$$

Hence,

$$
\omega^{(n+1)}\left(2, C_{n+1}\right) \leqq(1+\kappa)^{-1} \omega^{(n)}\left(2, C_{n}\right)
$$

and

$$
\omega(2, C) \leqq \lim _{n \rightarrow \infty} \omega^{(n)}\left(2, C_{n}\right)=0
$$

\section{REFERENCES}

1. L. Bers, Theory of Pseudo-analytic Functions, NYU, 1953.

2. A. Beurling and L. Ahlfors, The boundary correspondence under quasiconformal mappings, Acta Math., 96 (1976), 125-142.

3. A. Browder and J. Wermer, A method for constructing Dirichlet algebras, Proceedings Amer. Math. Soc., 15 (1964), 546-552.

4. A. Denjoy, Sur la continuité des fonctions analytiques singulières, Bull. Soc. Math. France, 60 (1932), 27-105.

5. P. Duren, Theory of $H^{p}$ Spaces, Academic Press, 1970.

6. K. Oikawa, Welding of polygons and the type of Riemann surfaces, Kōdai Math. Sem., 13 (1961), 37-52.

7. L. Volkoviskii, Quasiconformal mapping and the problem of conformal welding (Russian), Ukranski Mat. Ž., 2 (1951), 39-51.

Received April 14, 1975 and in revised form February 18, 1976. This work was supported in part by the N.S.F. under grant No. GP-42009.

University of CAlifornia, Los ANGeles 



\section{PACIFIC JOURNAL OF MATHEMATICS}

\section{EDITORS}

RICHARD ARENS (Managing Editor) University of California

Los Angeles, California 90024

R. A. Beaumont

University of Washington

Seattle, Washington 98105
J. DUGUNDJI

Department of Mathematics University of Southern Californı

Los Angeles, California 90007

D. Gilbarg and J. Milgram

Stanford University

Stanford, California 94305

\section{ASSOCIATE EDITORS}
E. F. BECKENBACH
B. H. NeumanN
F. WOLF
K. YosHIDA

\section{SUPPORTING INSTITUTIONS}

UNIVERSITY OF BRITISH COLUMBIA

CALIFORNIA INSTITUTE OF TECHNOLOGY

UNIVERSITY OF CALIFORNIA

MONTANA STATE UNIVERSITY

UNIVERSITY OF NEVADA

NEW MEXICO STATE UNIVERSITY

OREGON STATE UNIVERSITY

UNIVERSITY OF OREGON

OSAKA UNIVERSITY
UNIVERSITY OF SOUTHERN CALIFORNIA

STANFORD UNIVERSITY

UNIVERSITY OF TOKYO

UNIVERSITY OF UTAH

WASHINGTON STATE UNIVERSITY

UNIVERSITY OF WASHINGTON

* * * *

AMERICAN MATHEMATICAL SOCIETY

NAVAL WEAPONS CENTER 


\section{Pacific Journal of Mathematics \\ Vol. 65, No. $1 \quad$ September, 1976}

David Lee Armacost, Compactly cogenerated LCA groups ............. 1

Sun Man Chang, On continuous image averaging of probability measures ...... 13

J. Chidambaraswamy, Generalized Dedekind $\psi$-functions with respect to a

polynomial. II................................... 19

Freddy Delbaen, The Dunford-Pettis property for certain uniform algebras ..... 29

Robert Benjamin Feinberg, Faithful distributive modules over incidence

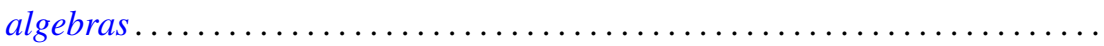

Paul Froeschl, Chained rings . . . . . . . . . . . . . . . . . . . .

John Brady Garnett and Anthony G. O'Farrell, Sobolev approximation by a sum

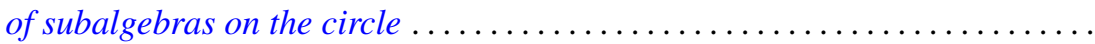

Hugh M. Hilden, José M. Montesinos and Thomas Lusk Thickstun, Closed

oriented 3-manifolds as 3-fold branched coverings of $S^{3}$ of special type.....

Atsushi Inoue, On a class of unbounded operator algebras ................

Peter Kleinschmidt, On facets with non-arbitrary shapes.

Narendrakumar Ramanlal Ladhawala, Absolute summability of Walsh-Fourier

series

Howard Wilson Lambert, Links which are unknottable by maps . . . . . . . . . . .

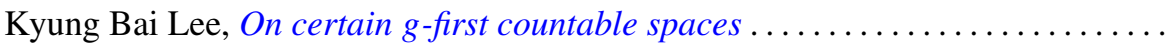

Richard Ira Loebl, A Hahn decomposition for linear maps .................

Moshe Marcus and Victor Julius Mizel, A characterization of functionals on $W_{1}^{p}$ possessing autonomous kernels. I . .

James Miller, Subordinating factor sequences and convex functions of several

variables.

Keith Pierce, Amalgamated sums of abelian l-groups ...

Jonathan Rosenberg, The $C^{*}$-algebras of some real and $p$-adic solvable

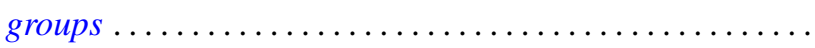

Hugo Rossi and Michele Vergne, Group representations on Hilbert spaces defined

in terms of $\partial_{b}$-cohomology on the Silov boundary of a Siegel domain . .

Mary Elizabeth Schaps, Nonsingular deformations of a determinantal

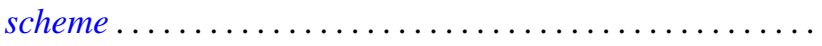

S. R. Singh, Some convergence properties of the Bubnov-Galerkin method...

Peggy Strait, Level crossing probabilities for a multi-parameter Brownian

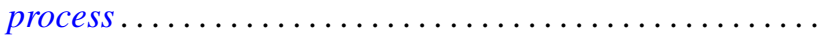

Robert M. Tardiff, Topologies for probabilistic metric spaces .

Benjamin Baxter Wells, Jr., Rearrangements of functions on the ring of integers of

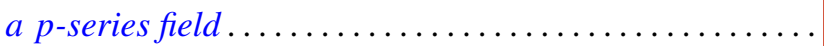

Robert Francis Wheeler, Well-behaved and totally bounded approximate identities for $C_{0}(X)$.

Delores Arletta Williams, Gauss sums and integral quadratic forms over local

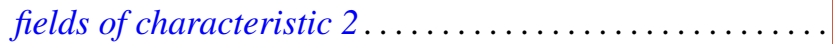

John Yuan, On the construction of one-parameter semigroups in topological 